IJMMS 2003:8, 461-474

PII. S0161171203208115

http://ijmms.hindawi.com

(c) Hindawi Publishing Corp.

\title{
ON FINITELY SUBADDITIVE OUTER MEASURES AND MODULARITY PROPERTIES
}

\author{
CHARLES TRAINA
}

Received 5 August 2002

\begin{abstract}
Let $v$ be a finite, finitely subadditive outer measure on $P(X)$. Define $\rho(E)=v(X)$ $v\left(E^{\prime}\right)$ for $E \subset X$. The measurable sets $S_{v}$ and $S_{\rho}$ and the set $S=\{E \subset X / v(E)=$ $\rho(E)\}$ are investigated in general, and in the presence of regularity or modularity assumptions on $v$. This is also done for $v^{0}(E)=\inf \left\{v(M) / E \subset M \in S_{v}\right\}$. General properties of $v$ are derived when $v$ is weakly submodular. Applications and numerous examples are given.
\end{abstract}

2000 Mathematics Subject Classification: 28A12, 28A10.

1. Introduction. Let $X$ be an arbitrary nonempty set and $v$ a finite, finitely subadditive outer measure on $P(X) . \rho$ denotes the set function defined by $\rho(E)=v(X)-v\left(E^{\prime}\right), E \subset X$. We investigate conditions for $\rho$ to be an inner measure. This entails the notion of weak submodularity for $v$, and we fully investigate the interplay between regularity, submodularity of $v$, and measurability of sets with respect to $v$. Such matters were first considered in [5], and, in this paper, we extend and improve upon the results. We also analyze a large number of examples. This can be done quickly in the light of our general results. In particular, we consider applications to well-known lattice derived outer measures.

We also apply our general results to the case of the finite, finitely subadditive outer measure $v^{0}(E)=\inf \left\{v(M) / E \subset M \in S_{v}\right\}, E \subset X$, where $S_{v}$ is the usual $\nu$-measurable sets. While this case is well known for $v$, a countably subadditive outer measure, it is not well known when $v$ is only finitely subadditive. Again, a start was made in [5], but we improve on these results and give much shorter proofs for known results.

We begin with a brief summary of the terminology and notation and some basic facts that will be used throughout the paper. We then consider the concepts of regularity, modularity, and condition (2.3) (see below) for an outer measure $v$ and establish their relationships. Next, examples are given, and then some interesting inequalities are established with applications. Finally, $\nu^{0}$ and the measurable sets $S_{v}$ and $S_{v^{0}}$ are considered with further examples presented. 
2. Background and notations. Throughout this section and the rest of the paper, $v$ will designate a finite-valued, finitely subadditive outer measure defined on the power set $P(X)$ of a nonempty set $X$. $\rho$ will designate the associated set function $\rho(E)=v(X)-v\left(E^{\prime}\right)$, where $E \subset X$, and the prime will designate complement. Clearly, $\rho(E) \leq \nu(E)$, for all $E \subset X$. $\rho$, in general, is not an inner measure (see Example 4.2). Furthermore, we let

$$
S=\{E \subset X / \rho(E)=\nu(E)\}
$$

and $S_{\nu}, S_{\rho}$ denote the $\nu$-measurable and $\rho$-measurable sets, respectively, where, in general, if $\lambda$ is a nonnegative real-valued set function on $P(X)$ with $\lambda(\phi)=0$, then we denote

$$
S_{\lambda}=\left\{E \subset X / \lambda(G)=\lambda(G \cap E)+\lambda\left(G \cap E^{\prime}\right) \forall G \subset X\right\} .
$$

It is well known that $S_{\lambda}$ is an algebra and $\lambda$ restricted to $S_{\lambda}$ is a finitely additive measure.

Again, let $v$ be a finite, finitely subadditive outer measure, then $v$ is regular if, for any $E \subset X$, there exists an $M \in S_{v}$ such that $E \subset M$ and $v(E)=v(M)$. If $v$ is just 0-1 valued, then, clearly, $v$ is regular. If $v$ is regular, then $E \in S_{v}$ if and only if

$$
v(X)=v(E)+v\left(E^{\prime}\right)
$$

(see [6]). Hence, we say that $v$ satisfies condition (2.3) if and only if $E \in S_{v}$ whenever $v(X)=v(E)+v\left(E^{\prime}\right)$. Therefore, we can say that any regular outer measure satisfies condition (2.3). The converse is false (see [6] and Section 4). We also apply this to $\rho$.

We can express these matters in terms of the above sets, $S_{v}$ and $S_{\rho}$. Clearly, $S_{v}$ and $S_{\rho}$ are contained in $S$, while $S=S_{v}$ if and only if condition (2.3) is satisfied for $\nu$, and $S=S_{\rho}$ if and only if condition (2.3) is satisfied by $\rho$. We note, for example, that if $v$ satisfies condition (2.3), then $S=S_{v}$ is an algebra. However, this is not true in general (see Section 4).

DEFINITION 2.1. Let $\mathscr{E}$ be a collection of subsets of $X$ such that $\mathscr{E}$ is a lattice, and let $\lambda$ be a set function defined on $\mathscr{E}$ such that

$$
\lambda(E \cup F)+\lambda(E \cap F) \leq \lambda(E)+\lambda(F)
$$

for all $E, F \in \mathscr{E}$, then $\lambda$ is submodular on $\mathscr{E}$. If the reverse inequality holds, then $\lambda$ is called supermodular on $\mathscr{E}$. It is called modular on $\mathscr{E}$ if we have equality. If $\mathscr{E}=P(X)$, we usually leave off the words "on $P(X)$," and simply say, for example, submodular. 
Next, let $\mathscr{C}$ be a covering class of $X$ such that $\phi \in \mathscr{C}$, and let $\tau$ be a finite, nonnegative set function defined on $\mathscr{C}$ with $\tau(\phi)=0$. Then, as well known,

$$
\lambda(E)=\inf \left\{\sum_{i=1}^{n} \tau\left(E_{i}\right) / E \subset \bigcup_{i=1}^{n} E_{i} \text {, for all } E_{i} \in \mathscr{b}\right\}
$$

is a finite and finitely subadditive outer measure on $P(X)$.

The following result (see [5]) is extremely useful especially for applications.

THEOREM 2.2. Let $\mathscr{C}, \tau$, and $\lambda$ be as above. Then,

(a) if $\mathscr{C}$ is closed under finite unions and if $\tau$ is finitely subadditive on $\mathscr{C}$, then

$$
\lambda(E)=\inf \{\lambda(C) / E \subset C \in \mathscr{C}\}, \quad \text { where } E \subset X ;
$$

if, in addition, $\tau$ is monotone, then $\lambda=\tau$ on $\mathscr{b}$;

(b) if $\mathscr{C}$ is a lattice and if $\tau$ is submodular on $\mathscr{C}$, then

$$
\lambda(E)=\inf \{\lambda(C) / E \subset C \in \mathscr{C}\}
$$

is submodular on $P(X)$.

Finally, let $\mathscr{L}$ be a lattice of subsets of $X$ such that $\phi, X \in \mathscr{L} . \mathscr{A}(\mathscr{L})$ denotes the algebra generated by $\mathscr{L}$, and $M(\mathscr{L})$ denotes the set of all nontrivial, nonnegative, finitely additive measures on $\mathscr{A}(\mathscr{L}) . M_{R}(\mathscr{L})$ denotes the set of all those $\mu \in$ $M(\mathscr{L})$ which are $\mathscr{L}$-regular. $M(\mathscr{L}), M_{R}(\mathscr{L})$, and other subsets of $M(\mathscr{L})$ have been extensively studied in the literature; we cite just a few recent papers $[1,4,7,8,9,10]$.

In Theorem 2.2, take $\mathscr{C}=\mathscr{L}^{\prime}$, the complementary lattice to $\mathscr{L}$, and $\tau=\mu \in$ $M(\mathscr{L})$. Then, writing $\mu^{\prime}$ for $\lambda$, we have the following theorem.

THEOREM 2.3. If $\mu \in M(\mathscr{L})$, then

$$
\mu^{\prime}(E)=\inf \left\{\mu\left(\mathscr{L}^{\prime}\right) / E \subset L^{\prime}, L \in \mathscr{L}\right\},
$$

for $E \subset X$, is a finite and finitely subadditive outer measure on $P(X)$ and $\mu^{\prime}$ is submodular on $P(X)$. Also,

$$
\rho(E)=\mu^{\prime}(X)-\mu^{\prime}\left(E^{\prime}\right)=\mu(X)-\mu^{\prime}\left(E^{\prime}\right)=\sup \{\mu(L) / L \subset E, L \in \mathscr{L}\}
$$

which has been denoted in the literature by $\mu_{i}(E)$.

We now proceed to extend and improve results in [5].

3. Modularity results. We again stress the fact that the finitely subadditive outer measure $v$ is always assumed to be finite valued. If this is not the case, then $\rho(E)=v(X)-v\left(E^{\prime}\right)$, for $E \subset X$, need not be defined. We have noted that 
$\rho$ need not be an inner measure (see Example 4.2). In order to see when $\rho$ is an inner measure, we introduce the following definition.

DEFINITION 3.1. Let $v$ be a finite and finitely subadditive outer measure. Then $v$ is weakly submodular if the submodular law

$$
v(E \cup F)+v(E \cap F) \leq v(E)+v(F),
$$

$E, F \subset X$, holds when $E \cup F=X$.

We now have the following theorem.

THEOREM 3.2. $\rho$ is an inner measure if and only if $v$ is weakly submodular.

PROof. Suppose that $v$ is a weakly submodular outer measure; all we must show is that

$$
\rho(E \cup F) \geq \rho(E)+\rho(F)
$$

if $E \cap F=\phi$. Now, since $E \cap F=\phi, E^{\prime} \cup F^{\prime}=X$. Therefore,

$$
v\left(E^{\prime} \cup F^{\prime}\right)+v\left(E^{\prime} \cap F^{\prime}\right) \leq v\left(E^{\prime}\right)+v\left(F^{\prime}\right) .
$$

Hence,

$$
v(X)-v\left(E^{\prime} \cup F^{\prime}\right)+v(X)-v\left(E^{\prime} \cap F^{\prime}\right) \geq v(X)-v\left(E^{\prime}\right)+v(X)-v\left(F^{\prime}\right) .
$$

That is,

$$
\rho(E \cup F) \geq \rho(E)+\rho(F) .
$$

The proof of the converse is just as simple.

We noted in Section 2 that the set

$$
S=\{E \subset X / \rho(E)=\nu(E)\}
$$

is not in general an algebra (see Example 4.4). However, we have the following theorem.

THEOREM 3.3. If $v$ is a finite, finitely subadditive outer measure and if $v$ is submodular, then $S$ is an algebra and $v$, restricted to $S$, is a finitely additive measure.

Proof. If $E \in S$, then, clearly, $E^{\prime} \in S$; while if $E, F \in S$, then, by the submodularity of $v$ which is of course equivalent to the supermodularity of $\rho$, we have

$$
\begin{aligned}
\rho(E)+\rho(F) & \leq \rho(E \cup F)+\rho(E \cap F) \\
& \leq v(E \cup F)+v(E \cap F) \\
& \leq v(E)+v(F)=\rho(E)+\rho(F) .
\end{aligned}
$$


This, together with the fact that $\rho \leq v$, implies that

$$
\rho(E \cup F)=v(E \cup F), \quad \rho(E \cap F)=v(E \cap F) .
$$

Hence, $S$ is an algebra. The last proof of the theorem follows now directly from (3.7).

We recall the following definition from [5].

DEFINITION 3.4. Let $v$ be, as usual, a finite, finitely subadditive outer measure, and let $S_{v}$ be the $v$-measurable sets. We define

$$
v^{0}=\inf \left\{v(M) / E \subset M \in S_{v}\right\}
$$

and say that $v$ is approximately regular if $v=v^{0}$.

It is clear from Theorem 2.2 that $v^{0}$ is a finite, finitely subadditive, submodular outer measure and $v^{0}(M)=v(M)$ for $M \in S_{v}$. We will consider $v^{0}$ in greater detail in Section 6.

In Section 2, we have noted that $v$ is regular if each $E \subset X$ has a measurable cover $M \in S_{v}$. Clearly, regularity implies approximately regular, and, in the case of $v$ being a countably subadditive outer measure where $S_{v}$ is a $\sigma$-algebra, the two notions coincide. However, this is not the case for finitely subadditive outer measures (see Section 4). The next theorem follows immediately from the definitions and the fact that $v$ is a finitely additive measure on $S_{v}$ and hence modular.

THEOREM 3.5. If $v$ is a finite, finitely subadditive outer measure, then $v$ is submodular if $v$ is approximately regular.

We now wish to investigate the relationship between modularity and condition (2.3) introduced in Section 2, and, in doing so, we will extend and improve results in [5].

THEOREM 3.6. (a) If $v$ is a finite, finitely subadditive outer measure, and if

$$
\rho(E)=v(X)-v\left(E^{\prime}\right) \quad \text { for } E \subset X,
$$

then $S_{v}=S_{\rho}$.

(b) If $v$ is also submodular on $P(X)$, then $S_{v}=S_{\rho}=S$.

Proof. (a) Let $E \in S_{v}$. Then, $v(A)=v(A \cap E)+v\left(A \cap E^{\prime}\right)$ for $A \subset X$. Now,

$$
\begin{aligned}
\rho(A \cap E) & =v(X)-v\left(A^{\prime} \cup E^{\prime}\right), \\
\rho\left(A \cap E^{\prime}\right) & =v(X)-v\left(A^{\prime} \cup E\right) .
\end{aligned}
$$

Therefore,

$$
\rho(A \cap E)+\rho\left(A \cap E^{\prime}\right)=2 v(X)-v\left(A^{\prime} \cup E^{\prime}\right)-v\left(A^{\prime} \cup E\right) .
$$


But

$$
\begin{gathered}
v\left(A^{\prime} \cup E\right)=v\left(\left(A^{\prime} \cup E\right) \cap E\right)+v\left(\left(A^{\prime} \cup E\right) \cap E^{\prime}\right)=v(E)+v\left(A^{\prime} \cap E^{\prime}\right), \\
v\left(A^{\prime} \cup E^{\prime}\right)=v\left(\left(A^{\prime} \cup E^{\prime}\right) \cap E\right)+v\left(\left(A^{\prime} \cup E^{\prime}\right) \cap E^{\prime}\right)=v\left(A^{\prime} \cap E\right)+v\left(E^{\prime}\right) .
\end{gathered}
$$

Substituting in (3.12), we have

$$
\begin{aligned}
\rho(A \cap E)+\rho\left(A \cap E^{\prime}\right) & =2 v(X)-v(E)-v\left(A^{\prime} \cap E^{\prime}\right)-v\left(E^{\prime}\right)-v\left(A^{\prime} \cap E\right) \\
& =v(X)-v\left(A^{\prime} \cap E\right)-v\left(A^{\prime} \cap E^{\prime}\right) \\
& =v(X)-v\left(A^{\prime}\right) \\
& =\rho(A) .
\end{aligned}
$$

Hence, $E \in S_{\rho}$, so $S_{v} \subset S_{\rho}$. Similarly, $S_{\rho} \subset S_{v}$. Thus, $S_{v}=S_{\rho} \subset S$.

(b) Since $v$ is submodular, $\rho$ is supermodular and, therefore, $S_{\rho}=S$ by [5]. We also see from part (a) that $\nu$ satisfies condition (2.3) if and only if $\rho$ satisfies condition (2.3).

We can summarize some results in the following theorem.

THEOREM 3.7. Let $v$ be a finite, finitely subadditive outer measure. Then, $v$ regular implies that $v$ is approximately regular implies that $v$ is submodular implies that $v$ satisfies condition (2.3).

We will see in the examples to follow that none of these implications can be reversed in general.

We also note that if $\mu$ is a countably additive measure or an algebra, then the customary induced Carathéodory outer measure $\mu^{*}$ is regular, and hence, if finite, it satisfies condition (2.3). Of course, this is well known [3, 6].

4. Examples. In view of our general results in Sections 2 and 3, many specific examples become easier to analyze. We first consider the important examples $\mu \in M(\mathscr{L})$ with $\nu=\mu^{\prime}$ and $\rho=\mu_{i}$ considered in Section 2. Using Theorems 2.3 and 3.7, we have the following theorem.

THEOREM 4.1. Let $\mu \in M(\mathscr{L})$ and

$$
\mu^{\prime}(E)=\inf \left\{\mu\left(L^{\prime}\right) / E \subset L^{\prime}, L \in \mathscr{L}\right\} .
$$

Then, $\mu^{\prime}$ is submodular, and the following are equivalent:

(a) $E \in S_{\mu^{\prime}}=S_{\mu_{i}}$,

(b) $\mu^{\prime}(E)=\mu_{i}(E)$ where $\mu_{i}(E)=\sup \{\mu(L) / L \subset E, L \in \mathscr{L}\}$,

(c) $\mu^{\prime}(E)+\mu^{\prime}\left(E^{\prime}\right)=\mu^{\prime}(X)$.

This result was first proved in [1] in an entirely different manner. It is, as we see, simply a special case of our more general theorems. It is also of interest to see when $\mu^{\prime}$ is even more than submodular, namely, approximately regular. We will look into this in Section 6. 
We now turn to more specific examples in order to show the need for certain hypotheses in the theorems and in order to show that certain implications cannot be reversed.

EXAMPLE 4.2. Let $X=\{1,2,3, \ldots\}$ and define $v(\phi)=0, v(X)=2, v(E)=1$ for all other sets $E \subset X$. Clearly, $v$ is an outer measure (countably subadditive), and $\rho(\phi)=0, \rho(X)=2, \rho(E)=1$ for all $E \subset X$. Clearly, $S=P(X)$ is of course an algebra, and, clearly, $S_{v}=\{\phi, X\}$. Thus, $v$ and $\rho$ do not satisfy condition (2.3); hence $v$ is not submodular and therefore not regular by Theorem 3.7. Also, even though $S$ is an algebra, $v$ is not a measure on $S$, which shows the need for submodularity in Theorem 3.3. Also, by taking any two disjoint nonempty sets whose union is not $\mathrm{X}$, we see that $\rho$ here is not an inner measure, so $v$ is not even weakly submodular. We finally note that, unlike two-valued (0-1) $v$, a three-valued $v$ need not be regular.

EXAmple 4.3. Again, let $X=\{1,2,3, \ldots\}$, and let $v(\phi)=0, v(X)=3 / 2$, $\nu(E)=1$ for all other $E \subset X$ (see [6]). Then, $\rho(\phi)=0, \rho(X)=3 / 2, \rho(E)=1 / 2$ for all other E. Clearly,

$$
S_{\rho}=S_{v}=S=\{\phi, X\}
$$

However, as easily seen, $v$ is not weakly submodular and, therefore, not submodular; yet, $v$ restricted to $S$ is trivially a measure. Also, condition (2.3) is of course true for $\nu$ and $\rho$.

EXAMPLE 4.4. Let $X=\{a, b, c, d\}$ and define $v(\phi)=0, v$ (any singleton) $=$ $1, v$ (any two-point set) $=1, v$ (any three-point set) $=2$, and $v(X)=2$. Then, $\rho(\phi)=0, \rho$ (singleton) $=0, \rho$ (a two-point set) $=1, \rho$ (a three-point set $)=1$, and $\rho(X)=2$. Hence,

$$
S=\{\phi, X, \text { all two-point sets }\}
$$

is not an algebra; therefore, $v$ and $\rho$ do not satisfy (2.3), so $v$ is not submodular, but $\rho$ is an inner measure, so $v$ is weakly submodular. Also, here, $S_{v}=S_{\rho}=$ $\{\phi, X\}$.

We have already proved or observed the following implications pertaining to the finite, finitely subadditive outer measure $v$

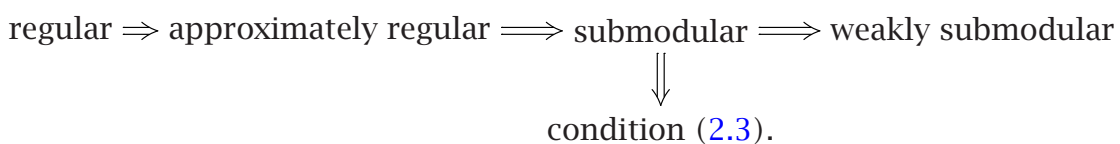

That weakly submodular does not imply submodular is shown in Example 4.4. That condition (2.3) does not imply submodular is shown in Example 4.3. We 
now give two type examples to show that submodularity does not imply approximately regular.

EXAMPLE 4.5. Let $X=\{a, b, c\}$ and define $v(\phi)=0$, and $v$ (singleton) $=1$, $\nu$ (any two-point set) $=2$, and $\nu(X)=2$. Then, $\rho(\phi)=0, \rho$ (singleton) $=0$, $\rho$ (a two-point set) $=1$, and $\rho(X)=2$. Thus, $S=\{\phi, X\}$. It is also routine to show that $v$ is submodular, and, hence, $v, \rho$ satisfy condition (2.3), so $S_{v}=S_{\rho}=$ $S=\{\phi, X\}$. However, this implies that $v^{0}$ just assumes the two values 0 or 2 . Consequently, $v \neq v^{0}$ and $v$ is not approximately regular which is equivalent to regular when $v$ just assumes a finite number of values.

Next, we consider the following example.

EXAMPLE 4.6. Let $X$ be an uncountable set. Define $v(\phi)=0=v$ (a finite set), $v$ (a countable set $)=1$, and $v$ (an uncountable set $)=2$. Then, $\rho(\phi)=$ $\rho$ (a finite set) $=0, \rho$ (a countable set $)=0$, and $\rho$ (an uncountable set $)=0,1$, and 2, depending on whether its complement is uncountable, countable, or finite. $v$ is easily seen to be submodular, and, clearly, $S=\{\phi, X$, all finite sets and their complements $\}$. Thus, $S_{\rho}=S_{v}=S$. Hence, $v^{0}$ just assumes the values 0 and 2; so, $v^{0} \neq v$ and $v$ is not approximately regular.

Finally, we consider an example for which $v$ is approximately regular but not regular. Clearly, $v$ must be finitely subadditive but not countably subadditive for such an example. Before proceeding to the example, we first recall that if $E \subset X$, then a measurable cover of $E$ is a set $M \in S_{v}$ such that $E \subset M$ and $v(M)=v(E)$. A measurable kernel of a set $E \subset X$ is a set $N \in S_{v}$ such that $N \subset E$ and $\rho(N)=\rho(E)$. Clearly, $E$ has a measurable cover if and only if $E^{\prime}$ has a measurable kernel.

Next, we note that if $\mathscr{A}$ is an algebra of subsets of $X$ and if $\mu$ is a finitely additive measure on $\mathscr{A}$, then

$$
\mu^{\cdot}(E)=\inf \{\mu(A) / E \subset A \in \mathscr{A}\}
$$

for $E \subset X$ is, by Theorem 2.2, a finitely subadditive outer measure on $P(X)$ which extends $\mu$ on $\mathscr{A}$ and $\mathscr{A} \subset S_{\mu}$. Also,

$$
\left(\mu^{\cdot}\right)^{0}(E)=\inf \left\{\mu^{\cdot}(B) / E \subset B \in S_{\mu^{\cdot}}\right\}=\mu^{\cdot}(E)
$$

for $E \subset X$; so, $\mu$ is approximately regular.

If $X=[0,1]$ and if $S \subset X$, we denote by $\bar{S}$ the closure of $S$ and by $S^{0}$ the interior of $S$.

EXAMPLE 4.7. Let $X=[0,1]$ and let $v=\bar{c}$, outer Jordan content. Then, $\rho=\underline{c}$, inner Jordan content. Also, let $0<\alpha<1$, and denote by $C_{\alpha}$ the Cantor set of measure $1-\alpha$. Although the Cantor set of measure zero is Jordan contentable, this is not the case for $C_{\alpha}$. Since $\underline{c}\left(C_{\alpha}^{\prime}\right)=\alpha$ while $\bar{c}\left(C_{\alpha}^{\prime}\right)=1-\underline{c}\left(C_{\alpha}\right)=1$ since $\underline{c}\left(C_{\alpha}\right)=\underline{c}\left(C_{\alpha}^{0}\right)=0$. 
It is clear by the above comments that $\bar{c}$ is approximately regular, and we now note that it is not regular. We show that $C_{\alpha}$ does not have a contentable cover or, equivalently, that $C_{\alpha}^{\prime}$ does not have a contentable kernel. Hence, suppose that there exists $S \subset C_{\alpha}^{\prime}, S \in S_{c}$, and

$$
\underline{c}(S)=\bar{c}(S)=\underline{c}\left(C_{\alpha}^{\prime}\right)=\alpha
$$

It readily follows that any open set containing an $x \in C_{\alpha}^{\prime}$ intersects $S$, so $C_{\alpha}^{\prime} \subset \bar{S}$. Consequently,

$$
[0,1]=\bar{C}_{\alpha}^{\prime} \subset \bar{S}
$$

since $C_{\alpha}^{0}=\phi$. Thus,

$$
\alpha=\bar{c}(S)=\bar{c}(\bar{S})=\bar{c}[0,1]=1,
$$

a contradiction. Hence, $C_{\alpha}^{\prime}$ does not have a contentable kernel, and $\bar{c}$ is not regular.

5. Further modularity properties. Again, $v$ denotes a finite, finitely subadditive outer measure on $P(X)$, and $\rho(E)=v(X)-v\left(E^{\prime}\right)$ for $E \subset X$. In this section, we prove a number of inequalities which significantly extend those in [5], and, in the course of proving these inequalities, we also give an alternate characterization of weakly submodular.

THEOREM 5.1. Let $v$ be a finite and finitely subadditive outer measure. Then, for $E, F \subset X$ and $E \cap F=\phi$,

(a) $\rho(E)+\rho(F) \leq \rho(E \cup F)$ if and only if $v$ is weakly submodular;

(b) $\rho(E \cup F) \leq \rho(E)+v(F)$;

(c) $\rho(E)+v(F) \leq v(E \cup F)$ if and only if $v$ is weakly submodular;

(d) $v(E \cup F) \leq v(E)+v(F)$.

Proof. (d) is of course true since $v$ is an outer measure. (a) was established in Theorem 3.2.

We proceed to prove (b). By definition of $\rho$ we have,

$$
\rho(E \cup F)=v(X)-v\left(E^{\prime} \cap F^{\prime}\right),
$$

but $E^{\prime}=F \cup\left(E^{\prime} \cap F^{\prime}\right)$, so $v\left(E^{\prime}\right) \leq v(F)+v\left(E^{\prime} \cap F^{\prime}\right)$. Thus, $v\left(E^{\prime}\right)-v(F) \leq$ $\nu\left(E^{\prime} \cap F^{\prime}\right)$, and, by (5.1), we get

$$
\rho(E \cup F) \leq v(X)-v\left(E^{\prime}\right)+v(F)=\rho(E)+\nu(F) .
$$

Finally we consider (c). Since $E \cap F=\phi, F^{\prime}=E \cup\left(F^{\prime} \cap E^{\prime}\right)$. Hence, $\rho\left(F^{\prime}\right) \geq$ $\rho(E)+\rho\left(F^{\prime} \cap E^{\prime}\right)$ since $v$ is weakly submodular. Now,

$$
\rho(E)+v(F)=v(X)+\rho(E)-\rho\left(F^{\prime}\right) \leq \mathcal{v}(X)-\rho\left(E^{\prime} \cap F^{\prime}\right)=\mathcal{v}(E \cup F) .
$$


Conversely, suppose that, whenever $A \cap B=\phi$,

$$
\rho(A)+v(B) \leq v(A \cup B) .
$$

Let $E \cap F=\phi$, so $F^{\prime}=E \cup\left(F^{\prime} \cap E^{\prime}\right)$. Taking $A=E$ and $B=F^{\prime} \cap E^{\prime}$, we get

$$
\rho(E)+v\left(F^{\prime} \cap E^{\prime}\right) \leq v\left(F^{\prime}\right)
$$

Therefore,

$$
\rho(E)+v\left(F^{\prime} \cap E^{\prime}\right) \leq v(X)-\rho(F) .
$$

So,

$$
\rho(E)+\rho(F) \leq v(X)-v\left(F^{\prime} \cap E^{\prime}\right)=\rho(E \cup F) .
$$

Hence, $\rho$ is an inner measure and $v$ is weakly submodular.

COROLLARY 5.2. If $v$ is a finite, finitely subadditive, and weakly submodular outer measure, then, for $E, F \subset X$, and $E \cap F=\phi$,

$$
\rho(E)+\rho(F) \leq \rho(E \cup F) \leq \rho(E)+\nu(F) \leq \nu(E \cup F) \leq v(E)+\mathcal{v}(F) .
$$

Corollary 5.2 is a strong extension of a result in [5], where it is only proven in the case of an approximately regular $\nu$. Since, as we noted in Theorem 2.3 for a $\mu \in M(\mathscr{L}), \mu^{\prime}$ is submodular, we have the following corollary.

COROllary 5.3. For $\mu \in M(\mathscr{L})$ and $E, F \subset X, E \cap F=\phi$,

$$
\mu_{i}(E)+\mu_{i}(F) \leq \mu_{i}(E \cup F) \leq \mu_{i}(E)+\mu^{\prime}(F) \leq \mu^{\prime}(E \cup F) \leq \mu^{\prime}(E)+\mu^{\prime}(F)
$$

COROLLARY 5.4. If $v$ is a finite, finitely subadditive, and weakly submodular outer measure, then, for $M \in S_{v}$ and $E \subset X$,

$$
\rho(M)=v(M)=\rho(M \cap E)+v\left(M \cap E^{\prime}\right) .
$$

Proof. By (b) and (c) of Theorem 5.1,

$$
\rho(M) \leq \rho(M \cap E)+v\left(M \cap E^{\prime}\right) \leq v(M)=\rho(M),
$$

which completes the proof.

There are clearly many special $\nu$, in addition to $\mu^{\prime}$, to which we can apply the above results. We will indeed consider one of these in Section 6. Further applications should be clear. 
6. The outer measure $v^{0}$. As usual, $v$ denotes a finite, finitely subadditive outer measure on $P(X)$. In Definition 3.4, we defined the finite, finitely subadditive outer measure

$$
v^{0}(E)=\inf \left\{\nu(M) / E \subset M \in S_{v}\right\} .
$$

Then,

$$
v^{0}(X)-v^{0}\left(E^{\prime}\right)=v(X)-v^{0}\left(E^{\prime}\right)=\sup \left\{v(M) / M \subset E, M \in S_{v}\right\}
$$

since $v^{0}=v$ on $S_{v} \subset S$, and we denote by $v_{0}$,

$$
v_{0}(E)=\sup \left\{v(M) / M \subset E, M \in S_{v}\right\} .
$$

Also, it is clear that

$$
v_{0} \leq \rho \leq v \leq v^{0}
$$

Clearly, these $v^{0}$ and $v_{0}$ fit the general framework of the previous sections. $v^{0}$ and $v_{0}$ have been thoroughly investigated in the case where $v$ is a countably subadditive outer measure (see [2]). They have also been investigated in the finitely subadditive case in [5]. In the light of our stronger results, we can improve on the results in [5] and also give considerably shorter proofs and numerous examples. We now proceed to do this.

The following theorem is proved in [5], and we add the proof for completeness.

THEOREM 6.1. Let $v$ be a finite and finitely subadditive outer measure. Then, $S_{v} \subset S_{v^{0}}$.

Proof. Let $E \in S_{v}$, and $A \subset X$ be arbitrary. For $\varepsilon>0$, there exists an $M \in S_{v}$ such that $A \subset M$ and $v(M)<v^{0}(A)+\varepsilon$. Now, $A \cap E \subset M \cap E \in S_{v}$, and $A \cap E^{\prime} \subset$ $M \cap E^{\prime} \in S_{v}$. Hence,

$$
v^{0}(A \cap E)+v^{0}\left(A \cap E^{\prime}\right) \leq v(M \cap E)+v\left(M \cap E^{\prime}\right)=v(M)<v^{0}(A)+\varepsilon,
$$

and, therefore, $E \in S_{v^{0}}$.

Next, we note that, for $E \subset X$,

$$
\begin{aligned}
v^{00}(E) & =\inf \left\{v^{0}(M) / E \subset M \in S_{v_{0}}\right\} \\
& \leq \inf \left\{v^{0}(M) / E \subset M \in S_{v}\right\},
\end{aligned}
$$

by Theorem 6.1 , and this, in turn,

$$
\begin{aligned}
& =\inf \left\{v(M) / E \subset M \in S_{v}\right\} \\
& =v^{0}(E) ;
\end{aligned}
$$


so, $v^{00} \leq v^{0}$. But we always have $v^{00} \geq v^{0}$. Consequently, $v^{00}=v^{0}$; so, $v^{0}$ is approximately regular, and we can use the results of the preceding sections on $v^{0}$.

THEOREM 6.2. Let $v$ be a finite, finitely subadditive outer measure. Then,

(a) $v^{0}$ is approximately regular and hence submodular and satisfies condition (2.3);

(b) $S_{\rho}=S_{v} \subset S_{v^{0}}=S_{v_{0}}=\tilde{S} \subset S$, where $\tilde{S}=\left\{E \subset X / \nu_{0}(E)=v^{0}(E)\right\}$;

(c) if $E, F \subset X$ and $E \cap F=\phi$, then

$v_{0}(E)+v_{0}(F) \leq v_{0}(E \cup F) \leq v_{0}(E)+v^{0}(F) \leq v^{0}(E \cup F) \leq v^{0}(E)+v^{0}(F) ;$

(d) for $M \in S_{v_{0}}$ and $E \subset X$,

$$
v_{0}(M)=v^{0}(M)=v_{0}(M \cap E)+v^{0}\left(M \cap E^{\prime}\right) .
$$

Proof. (a) Since we have seen that $v^{0}$ is approximately regular, (a) follows from Theorem 3.7. (b) follows from Theorems 3.6 and 6.1(a), and since

$$
v_{0}(E) \leq \rho(E) \leq v(E) \leq v^{0}(E),
$$

it follows that $E \in \tilde{S}$ implies $E \in S$.

COROLLARY 6.3. If $v$ is a finite, finitely subadditive outer measure that satisfies condition (2.3), then $S_{\rho}=S_{v}=S_{v_{0}}=S_{v^{0}}=\tilde{S}=S$.

PROoF. The proof follows immediately from Theorem 6.2(b), and since $v$ satisfies condition (2.3), $S_{v}=S$.

REMARK 6.4. We have shown that $S_{v} \subset S_{v^{0}}$ and that $S_{v}=S_{v^{0}}$ if $v$ satisfies condition (2.3). It is certainly possible that $S_{v}=S_{v^{0}}$ even if condition (2.3) is not satisfied (see examples below).

We do have, however, the following result.

THEOREM 6.5. Let $E \in S_{v^{0}}$, then $E \in S_{v}$ if and only if there exists an $M \subset E$, $M \in S_{v}$, and $v(E)=v(M)$.

Proof. If the condition holds, then

$$
v(E)=v(E \cap M)+v\left(E \cap M^{\prime}\right)=v(M)+v\left(E \cap M^{\prime}\right) .
$$

Hence, $v\left(E \cap M^{\prime}\right)=0$, so, $E \cap M^{\prime} \in S_{v}$, and, therefore,

$$
E=M \cup\left(E \cap M^{\prime}\right) \in S_{v} .
$$

The converse is of course clear.

REMARK 6.6. In the case of a countably subadditive measure $v$ which is finite, the condition in Theorem 6.5 is satisfied, and we always have $S_{v}=S_{v^{0}}$. 
However, if $v$ can take on infinite values, then this is no longer the case. As noted earlier, this is treated in full detail in [2].

COROLLARY 6.7. If $v$ is a finite, finitely subadditive outer measure and if

(a) $v$ just assumes a finite number of values on $S_{v}$ or

(b) $S_{v}$ is a $\sigma$-algebra, then $S_{v}=S_{v^{0}}$.

Proof. In both cases, it is clear that the condition of Theorem 6.5 is satisfied.

We now consider several examples.

EXAMPLE 6.8. We first consider the general example of a $\mu \in M(\mathscr{L})$. Since $\mu^{\prime}$ is submodular, we have, by Corollary $6.3, S_{\mu^{\prime}}=S_{\left(\mu^{\prime}\right)}$. Suppose next that $\mu \in M_{R}(\mathscr{L})$. This is equivalent to $\mathscr{L} \subset S_{\mu^{\prime}}$. Hence,

$$
\begin{aligned}
\mu^{\prime}(E) & =\inf \left\{\mu\left(L^{\prime}\right) / E \subset L^{\prime} \in \mathscr{L}^{\prime}\right\} \\
& \geq \inf \left\{\mu^{\prime}(M) / E \subset M \in S_{\mu^{\prime}}\right\} \\
& =\left(\mu^{\prime}\right)^{0}(E) \geq \mu^{\prime}(E) .
\end{aligned}
$$

Thus, we have $\mu \in M_{R}(\mathscr{L})$ that implies that $\left(\mu^{\prime}\right)^{0}=\mu^{\prime}$, that is, $\mu^{\prime}$ is approximately regular. The converse need not be true since, for any $\mu \in M(\mathscr{L})$ that just assumes two values, $\mu^{\prime}$ is regular.

We next consider two specific examples.

EXAMPLE 6.9. Let $X, v$ be as in Example 4.6. We saw that $v$ is submodular. Hence, by Corollary 6.3, $S_{v}=S_{v_{0}}$. This also follows by Corollary 6.7. We also note that $v$ is just finitely subadditive here.

EXAMPLE 6.10. Let $X=\{1,2,3, \ldots\}$; and define $v(\phi)=0, v$ (a finite set) $=0$, $v(E)=1$ (if $E$ is infinite and $E^{\prime}$ is infinite), and $v(E)=2$ (if $E$ is infinite and $E^{\prime}$ is finite), so, $\nu(X)=2$. Then, $\rho(\phi)=0, \rho$ (a finite set) $=0, \rho(E)=1$ (if $E$ is infinite and $E^{\prime}$ is infinite), and $\rho(E)=2$ (if $E$ is infinite and $E^{\prime}$ is finite). Thus, $S=P(X)$ while $S_{v}=\left\{\phi, X, E\right.$ such that $E$ or $E^{\prime}$ is finite $\}$. Thus, $S \neq S_{v}$; so, condition (2.3) is not satisfied here but by Corollary $6.7 S_{v}=S_{v^{0}}$. Thus, $S_{v}=S_{v^{0}}$ does not imply condition (2.3). We also note that $v$ here is clearly only finitely subadditive.

Different examples in the case of a finite, finitely subadditive outer measure of a general type can be found in [5].

\section{REFERENCES}

[1] P. M. Grassi, Outer measures and associated lattice properties, Int. J. Math. Math. Sci. 16 (1993), no. 4, 687-694.

[2] H. Hahn and A. Rosenthal, Set Functions, The University of New Mexico Press, New Mexico, 1948. 
[3] P. R. Halmos, Measure Theory, Van Nostrand, New York, 1950.

[4] P.-S. Hsu, Characterizations of outer measures associated with lattice measures, Int. J. Math. Math. Sci. 24 (2000), no. 4, 237-249.

[5] J. E. Knight, Some remarks concerning finitely subadditive outer measures with applications, Int. J. Math. Math. Sci. 21 (1998), no. 4, 653-669.

[6] M. E. Munroe, Introduction to Measure and Integration, Addison-Wesley Mathematics Series, Addison-Wesley, Massachusetts, 1953.

[7] J. Ponnley, On lattice associated outer measures and applications to smoothness conditions, J. Math. Sci. (Calcutta) 9 (1998), no. 1, 57-69.

[8] C. Traina, Outer measures associated with lattice measures and their application, Int. J. Math. Math. Sci. 18 (1995), no. 4, 725-734.

[9] _ L L Lattice related outer measure inequalities on pairs of lattices, J. Math. Sci. (Calcutta) 12 (2001), no. 2, 53-69.

[10] S. Wibisono, Smoothness, regularity, and domination of lattice measures, J. Math. Sci. (Calcutta) 6 (1995), no. 2, 47-60.

Charles Traina: Department of Mathematics and Computer Science, St. John's University, 8000 Utopia Parkway, Jamaica, NY 11439, USA

E-mail address: trainac@stjohns.edu 


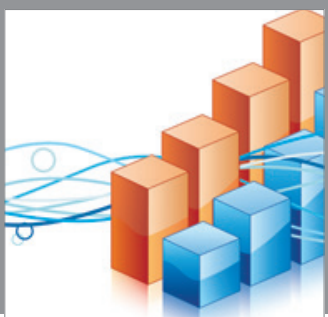

Advances in

Operations Research

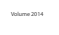

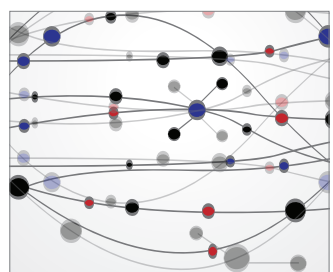

\section{The Scientific} World Journal
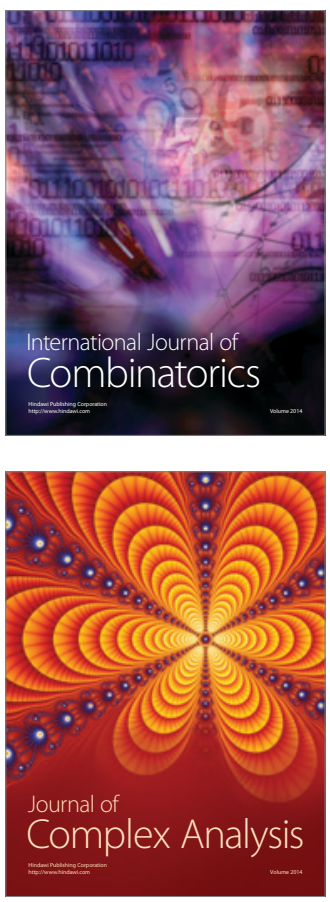

International Journal of

Mathematics and

Mathematical

Sciences
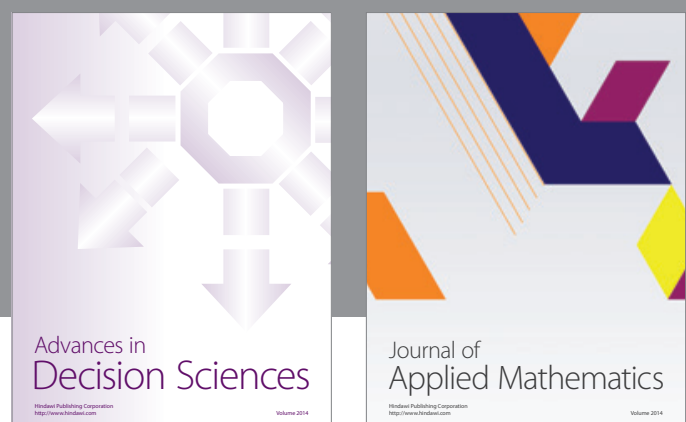

Journal of

Applied Mathematics
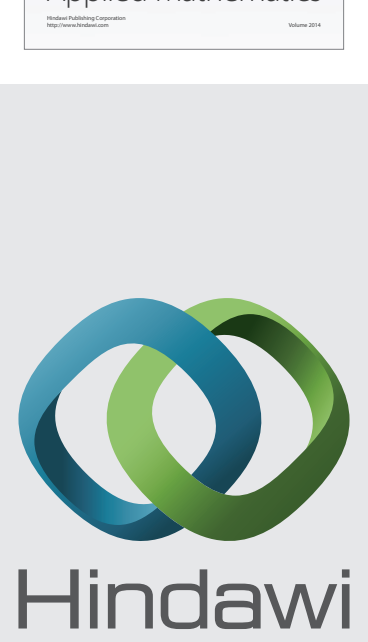

Submit your manuscripts at http://www.hindawi.com
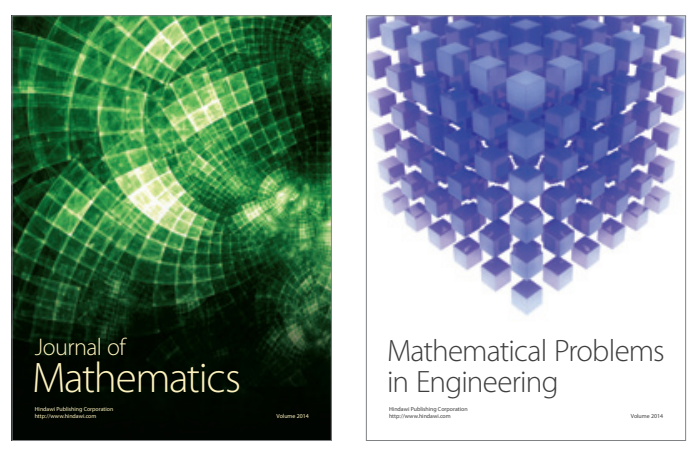

Mathematical Problems in Engineering
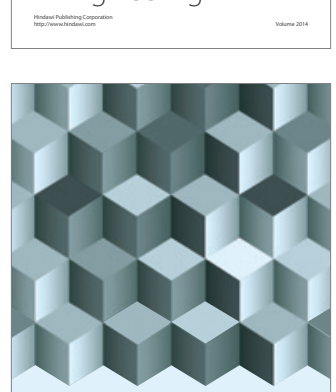

Journal of

Function Spaces
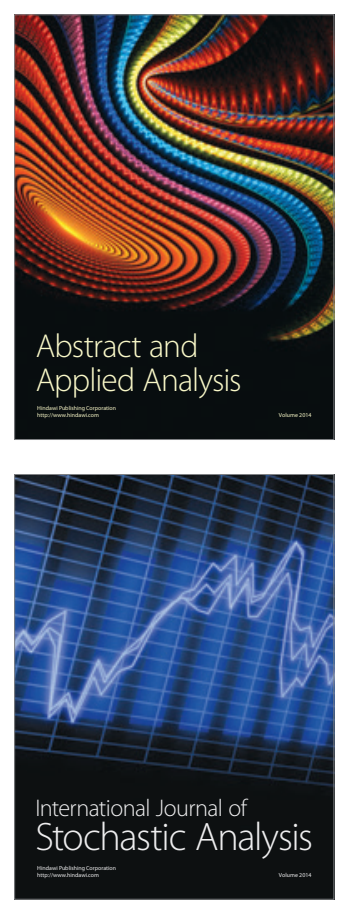

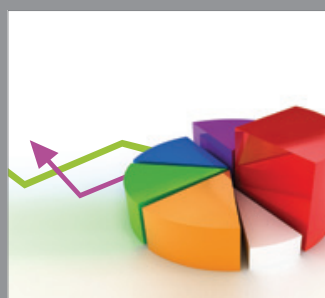

ournal of

Probability and Statistics

Promensencen
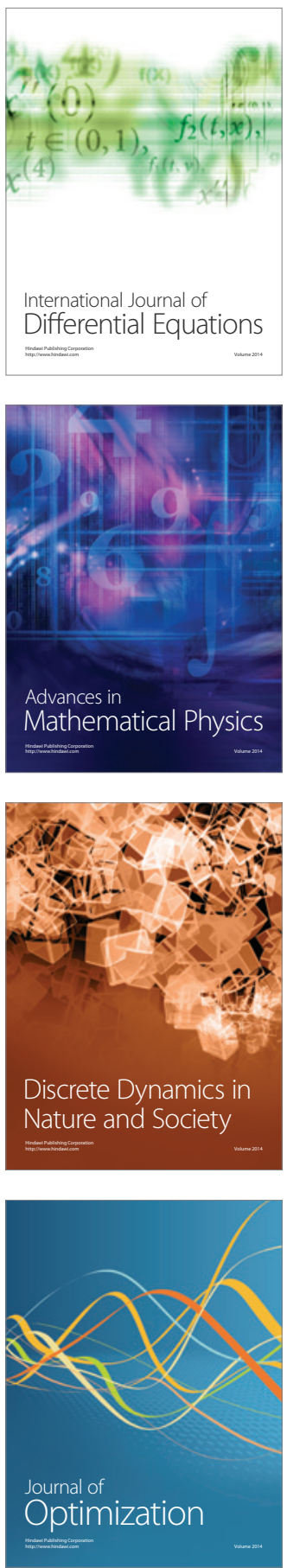